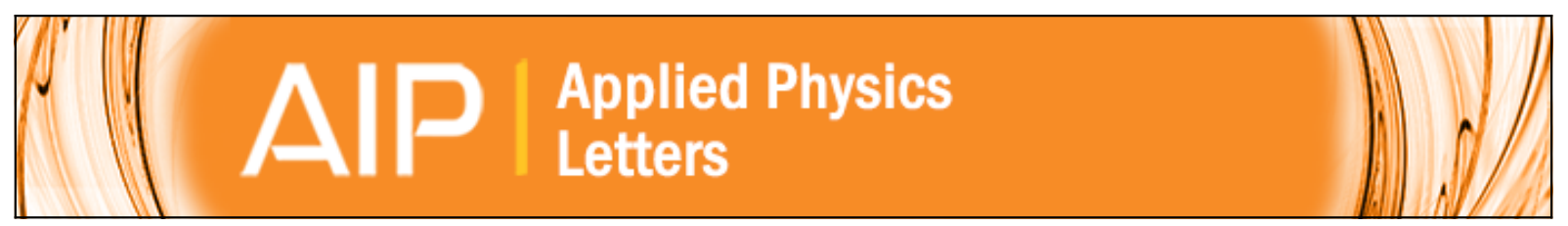

\title{
Boundary between the thermal and statistical polarization regimes in a nuclear spin
} ensemble

B. E. Herzog, D. Cadeddu, F. Xue, P. Peddibhotla, and M. Poggio

Citation: Applied Physics Letters 105, 043112 (2014); doi: 10.1063/1.4892361

View online: http://dx.doi.org/10.1063/1.4892361

View Table of Contents: http://scitation.aip.org/content/aip/journal/apl/105/4?ver=pdfcov

Published by the AIP Publishing

\section{Articles you may be interested in}

Observation of strongly forbidden solid effect dynamic nuclear polarization transitions via electron-electron double resonance detected NMR

J. Chem. Phys. 139, 214201 (2013); 10.1063/1.4832323

Analysis of the Ammonia Target Polarization

AIP Conf. Proc. 980, 322 (2008); 10.1063/1.2888104

Ultrafast Nuclear Spin Polarization by Short Laser Pulses

AIP Conf. Proc. 980, 289 (2008); 10.1063/1.2888099

Nuclear spin polarization induced by ultrashort laser pulses

AIP Conf. Proc. 915, 996 (2007); 10.1063/1.2750942

Nuclear Spin Polarizer for SolidState NMR Quantum Computers

AIP Conf. Proc. 772, 1471 (2005); 10.1063/1.1994671

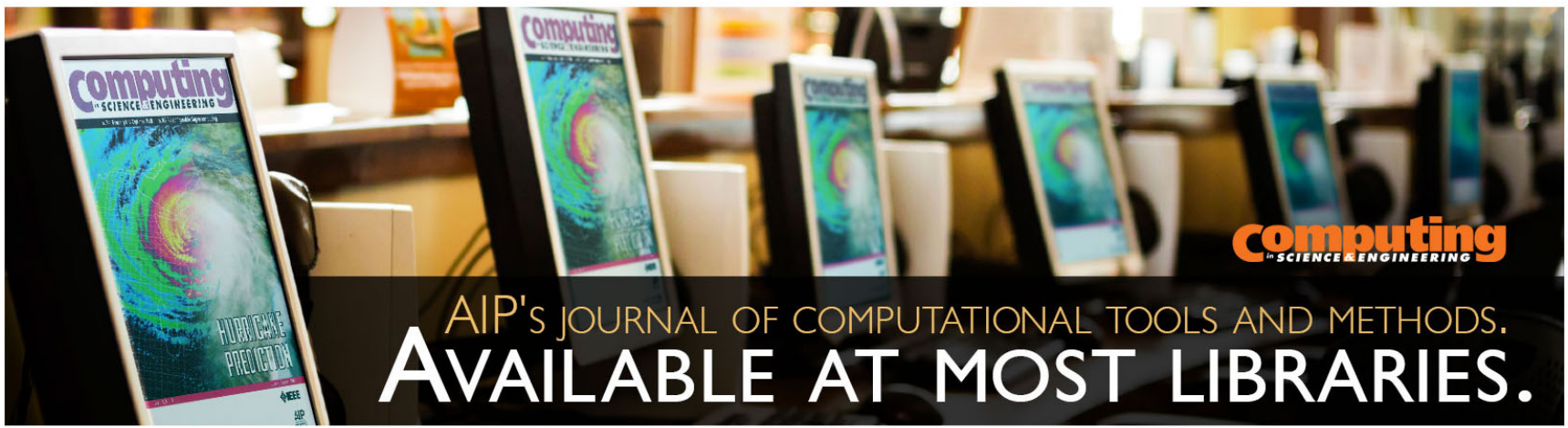




\title{
Boundary between the thermal and statistical polarization regimes in a nuclear spin ensemble
}

\author{
B. E. Herzog, D. Cadeddu, F. Xue, P. Peddibhotla, and M. Poggio a) \\ Department of Physics, University of Basel, Klingelbergstrasse 82, 4056 Basel, Switzerland
}

(Received 24 June 2014; accepted 22 July 2014; published online 1 August 2014)

\begin{abstract}
As the number of spins in an ensemble is reduced, the statistical fluctuations in its polarization eventually exceed the mean thermal polarization. This transition has now been surpassed in a number of recent nuclear magnetic resonance experiments, which achieve nanometer-scale detection volumes. Here, we measure nanometer-scale ensembles of nuclear spins in a $\mathrm{KPF}_{6}$ sample using magnetic resonance force microscopy. In particular, we investigate the transition between regimes dominated by thermal and statistical nuclear polarization. The ratio between the two types of polarization provides a measure of the number of spins in the detected ensemble. C 2014 AIP Publishing LLC.

[http://dx.doi.org/10.1063/1.4892361]
\end{abstract}

In recent decades, the drive for technological advancement coupled with an interest in understanding underlying microscopic interactions has led to rapid growth in the number of studies related to nanometer-scale phenomena. The research area broadly known as nanoscience and nanotechnology brings together a diverse range of topics including surface science, semiconductor physics, and molecular selfassembly. In many systems, physical phenomena at the nanometer-scale are strikingly different from their behavior at the macroscale. In particular, the reduced dimensionality of nanometer-scale samples can manifest itself in either thermal or quantum effects not observed in larger systems. For example, behavior ranging from the Brownian motion ${ }^{1}$ to the quantization of conductance ${ }^{2}$ emerges as measurement length scales are reduced.

The development of nuclear magnetic resonance (NMR) and magnetic resonance imaging (MRI) with nanometer-scale resolution has been a particularly captivating goal in nanoscience, due to its potential impact. As the only nondestructive, chemically selective, and truly three-dimensional imaging technique, MRI is an indispensable tool in a broad array of fields including medicine, biology, physics, and materials science. Conventional inductively detected MRI, however, is limited to a detection volume of a few $\mu \mathrm{m}^{3}{ }^{3}$. The extension of this resolution down to a few $\mathrm{nm}^{3}$ and eventually to atomic resolution has been a long-standing goal. ${ }^{4}$ The capability to image molecules atom-by-atom, thus allowing the mapping of the three-dimensional atomic structure of unknown macromolecules would be revolutionary. While the latter goal has not yet been achieved, a few experiments in the last few years have demonstrated nanometer-scale MRI (nanoMRI). ${ }^{5,6}$ Two techniques, magnetic resonance force microscopy (MRFM) first, and nitrogen-vacancy (NV) magnetometry shortly thereafter, have both detected NMR in nanometer-scale detection volumes. ${ }^{8-10}$ Although so far only MRFM techniques have produced 3D images of nuclear spin density, e.g., virus particles and hydrocarbon layers, ${ }^{5-7} \mathrm{NV}$ magnetometry has achieved a higher sensitivity $^{11}$ and initial imaging experiments ${ }^{12-14}$ have recently been made. In addition, NV magnetometry appears particularly

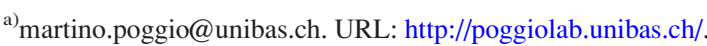

promising given its ability to work under ambient conditions, while high-sensitivity MRFM must be carried out in high vacuum and at cryogenic temperatures.

Nanometer-scale spin ensembles differ from larger ensembles in that random fluctuations in the total polarization-also known as spin noise-exceed the normally dominant mean thermal polarization. This characteristic imposes important differences between nanoMRI and conventional MRI protocols. In the former technique, statistical fluctuations are usually measured, whereas in the latter the signal is based on the thermal polarization. ${ }^{15-17}$ The thermal polarization-also known as Boltzmann polarization-results from the alignment of nuclear magnetization under thermal equilibrium along a magnetic field. The statistical polarization, on the other hand, arises from the incomplete cancellation of magnetic moments within the ensemble. Here, we study the nuclear polarization of nanometer-scale volumes using MRFM, focusing on the transition between the regimes in which thermal and statistical polarization dominate.

A single spin in a magnetic field can be described by the Hamiltonian $\hat{H}=-\hbar \gamma B \hat{I}_{z}$, where $\hbar$ is the reduced Plank constant, $\gamma$ is the gyromagnetic ratio, $B$ is the total magnetic field, and $\hat{I}_{z}$ is the nuclear spin operator along $\hat{z}$. For an ensemble of $N$ non-interacting spins, we calculate the expectation value for $M_{z}$, i.e., the mean magnetization along $\hat{z}$, as well as its standard deviation $\sigma_{M_{z}}$ using the partition function and density matrix. ${ }^{18,19}$ Considering that the thermal energy even at cryogenic temperatures $(T \sim 1 \mathrm{~K})$ and high magnetic fields $(B \sim 10 \mathrm{~T})$ is much larger than the nuclear Zeeman splitting, i.e., $\hbar \gamma B \ll k_{B} T$, we neglect orders of $\frac{\hbar \gamma B}{k_{B} T}$ beyond the first, resulting in

$$
\begin{gathered}
M_{z}=N \frac{I(I+1)}{3}\left(\frac{\hbar \gamma B}{k_{B} T}\right) \hbar \gamma, \\
\sigma_{M_{z}}=\sqrt{N \frac{I(I+1)}{3} \hbar \gamma,}
\end{gathered}
$$

where $k_{B}$ is the Boltzmann constant and $T$ is the temperature of the system.

In order to compare the thermal and the statistical polarization, we express both as fractions of a fully polarized 
system $M_{100 \%}=N \hbar \gamma I$, resulting in $P_{\text {thermal }}=\frac{M_{z}}{M_{100 \%}}=\frac{I+1}{3} \frac{\hbar \gamma B}{k_{B} T}$ and $P_{\text {statistical }}=\frac{\sigma_{M_{z}}}{M_{100 \%}}=\sqrt{\frac{I+1}{3 I} \frac{1}{N}}$. Note that while $P_{\text {thermal }}$ is independent of the ensemble size, $P_{\text {statistical }}$ increases with decreasing ensemble size. This implies that for ensembles with $N<N_{c}$, where $N_{c}$ is some critical number of spins reflecting the border of the two regimes, $P_{\text {statistical }}>P_{\text {thermal }}$. For this ensemble size, the size of the natural spin polarization fluctuations will begin to exceed the magnitude of the mean polarization in thermal equilibrium. This transition typically occurs on the micro- or nanometer-scale, underpinning the dominant role that statistical fluctuations play in nanometer-scale NMR. Furthermore, by measuring both mean thermal magnetization (1) and the standard deviation (2), one can determine the number of spins in the detected ensemble depending on the ratio of $M_{z}$ and $\sigma_{M_{z}}$

$$
N=\frac{3}{I(I+1)}\left(\frac{k_{B} T}{\hbar \gamma B}\right)^{2}\left(\frac{M_{z}}{\sigma_{M_{z}}}\right)^{2} .
$$

Note that for $\frac{M_{z}}{\sigma_{M_{z}}}=1$, the ensemble contains $N=N_{c}$ spins. In a material with a nuclear spin density $n a$, where $n$ is the number density of the nuclear element and $a$ is the natural abundance of the measured isotope, the corresponding detection volume is then given by $V=\frac{N}{n a}$.

We measure nanometer-scale volumes of ${ }^{19} \mathrm{~F}$ spins in a sample of $\mathrm{KPF}_{6}$ by MRFM. The $(1.2 \times 1.4 \times 3.2)-\mu \mathrm{m}^{3}$ sample is glued to the end of an ultra-sensitive $\mathrm{Si}$ cantilever. The cantilever is $130-\mu \mathrm{m}$-long, 4- $\mu \mathrm{m}$-wide, $0.1-\mu \mathrm{m}$-thick, and has a spring constant $k=75 \mu \mathrm{N} / \mathrm{m}$, as determined by thermal noise measurements at various temperatures. In the cryogenic measurement chamber at $T=4.4 \mathrm{~K}$ and in a vacuum better than $10^{-6}$ mbar, the sample-loaded cantilever has a mechanical resonance frequency $f_{c}=3.28 \mathrm{kHz}$ and a quality factor $Q=3.1 \times 10^{4}$. The apparatus includes a fiber-optic interferometer to measure the cantilever's displacement $x$ and a superconducting magnet for the application of an external field up to $B_{\text {ext }}=6 \mathrm{~T}$ along the cantilever axis $\hat{z}$. Immediately beneath the sample - at a distance of $50-100 \mathrm{~nm}$ - a nanomagnetic tip integrated on top of a metallic microwire produces strong spatial magnetic field gradients, as shown in Fig. 1. ${ }^{20}$ To reduce electrostatic interactions between the magnetic tip and the sample, a 15-nm-thick layer of $\mathrm{Au}$ is evaporated on the sample after attachment.

The microwire acts as a radio frequency source for the application of adiabatic rapid passage (ARP) pulses of the transverse field to the spin ensemble. ${ }^{21,22}$ We drive current through the microwire with the frequency-sweep waveform shown in Fig. 1(b). By synchronizing the ARP pulses such that they produce a transverse rf magnetic field whose frequency is swept through the nuclear magnetic resonance twice every cantilever period, $1 / f_{c}=T_{c}$, we drive longitudinal nuclear spin flips in the sample at $f_{c}$. Since the sample is affixed to the end of the cantilever, in the presence of the

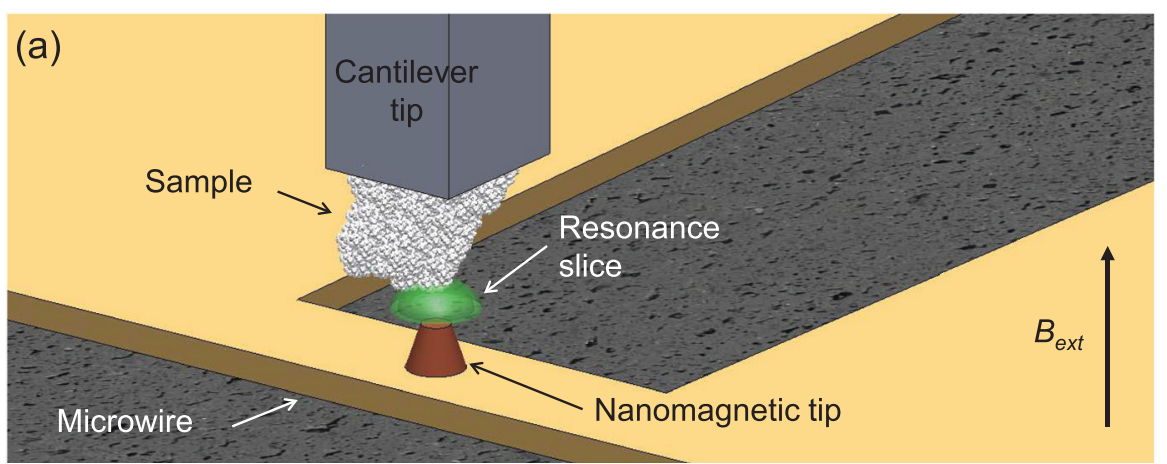

(b)

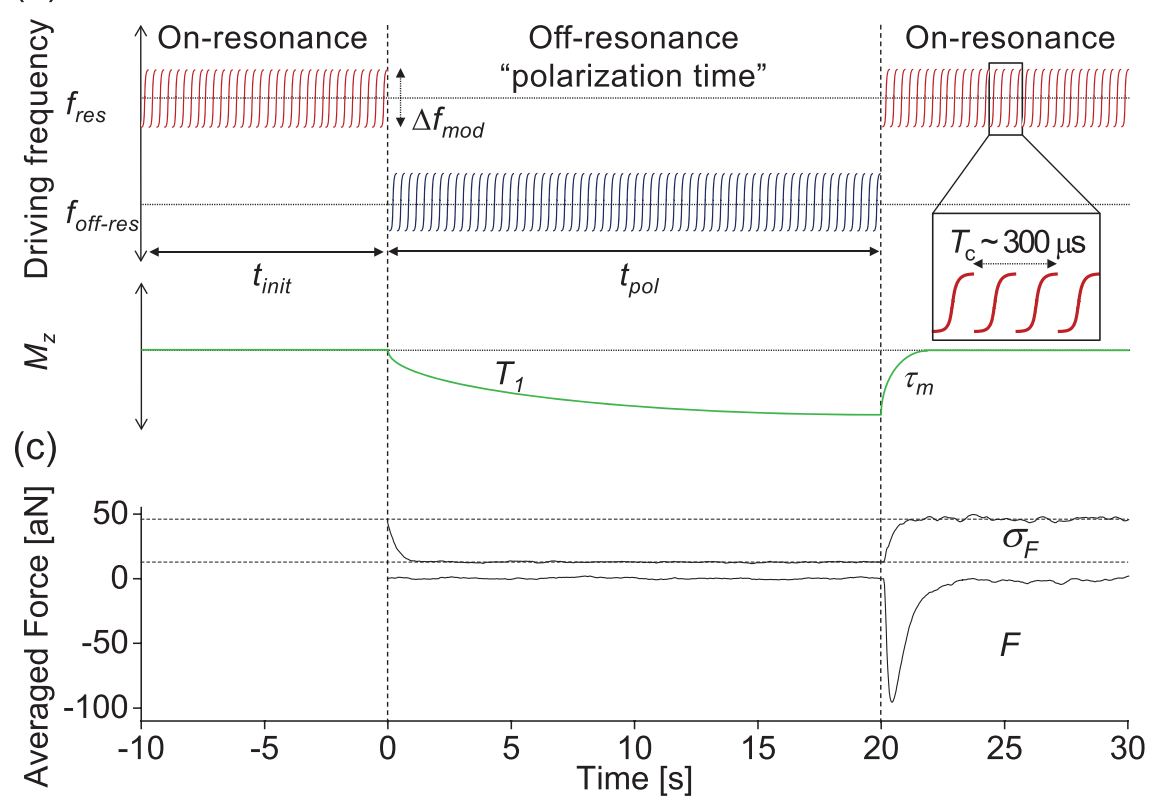

FIG. 1. (a) The experimental setup with the poly crystalline $\mathrm{KPF}_{6}$ sample (white) at the end of the cantilever. A small section of it intersects with the resonance slice (green) above the nanomagnet. The microwire produces the transverse rf magnetic field used to adiabatically invert the nuclear spins. (b) Schematic diagram of the pulse sequence and the response of the average nuclear magnetization $M_{z}$. The pulse spacing has been exaggerated for clarity. (c) $F$ and $\sigma_{F}$ averaged over 500 measurements for $t_{\mathrm{pol}}=20 \mathrm{~s}$ and $\Delta f=3 \mathrm{MHz}$. The fast decay of $F$ after the pulses are switched on resonance is due to the correlation time of the spins during the ARP pulses, $\tau_{m}$. 
large magnetic field gradient $\frac{\partial B}{\partial x}$ generated by the nanomagnetic tip, the spin flips produce an alternating force that drives the cantilever's mechanical resonance. By measuring the amplitude of the cantilever's resonant oscillations $x(t)$ with the fiber interferometer and a lock-in amplifier, we therefore determine the force acting on it $F(t)=\frac{k}{Q} x(t)$. Note that during the measurement, we damp the cantilever using electronic feedback to a quality factor $Q=400$ in order to increase the bandwidth $\Delta f_{\text {meas }}$ of our force detection without sacrificing force sensitivity. ${ }^{20}$ From $F(t)$, we derive the average force $F$ and the its standard deviation $\sigma_{F}$ over a fixed time interval.

The volume of spins, which cyclically inverts at $f_{c}$ due to the ARP pulses, is known as the resonant slice. The position and volume of this slice is determined by the spatial dependence of the magnetic field produced by the nanomagnetic tip $B_{\text {tip }}$ and by the parameters of the pulses. A schematic representation of the resonant slice, the nanomagnetic tip, and the ARP pulse sequence are shown in Fig. 1. The intersection of the resonant slice with the sample constitutes the volume of spins addressed by the ARP pulses and therefore the NMR detection volume $V$. Given the parameters used in these experiments, $V$ is always concentrated to a small region of space less than $(100 \mathrm{~nm})^{3}$.

When the ARP pulses are tuned to the NMR frequency of nuclei inside the resonant slice, the mean and standard deviation of the force acting on the cantilever, $F$, and $\sigma_{F}$, respectively, depend on the mean and standard deviation of the spin ensemble's magnetization, $M_{z}$, and $\sigma_{M_{z}}: F=\frac{\partial B}{\partial x} M_{z}$ and $\sigma_{F}=\sqrt{\sigma_{\text {spin }}^{2}+\sigma_{\text {cant }}^{2}}=\sqrt{\left(\frac{\partial B}{\partial x}\right)^{2} \sigma_{M_{z}}^{2}+\sigma_{\text {cant }}^{2}}$, where $B=B_{\text {ext }}+B_{\text {tip }}$ is the total magnetic field in the detection volume, ${ }^{23} \hat{x}$ is the direction of the cantilever oscillation, and by the fluctuation-dissipation theorem $\sigma_{\text {cant }}=\sqrt{\frac{2 k k_{B} T \Delta f_{\text {meas }}}{\pi f_{c} Q}}$ is the standard deviation of the random thermal force acting on the cantilever (the lock-in bandwidth $\Delta f_{\text {meas }}=0.5 \mathrm{~Hz}$ must fulfill $\Delta f_{\text {meas }}<f_{c} / Q$ for the damped $Q=400$ ). Off resonance, only thermal fluctuations drive the cantilever resulting in $F=0$ and $\sigma_{F}=\sigma_{\text {cant }}$.

In order to measure the size of the thermal nuclear polarization, we first initialize the spins to a mean polarization of zero by applying the ARP pulse sequence with its carrier frequency set to $f_{\text {res }}$, the NMR frequency of the nuclear spins of interest. During the application of the resonant ARP pulses, the nuclear spins have a short correlation time $\tau_{m} \approx 200 \mathrm{~ms}$. Therefore, by applying resonant pulses for a time $t_{\text {init }} \gg \tau_{m}$, the initial thermal polarization is erased, leaving only the statistical polarization fluctuations. $\tau_{m} \ll T_{1}$ and is limited by the relaxation time in the rotating frame $T_{1 \rho}$ and the adiabaticity of the ARP pulses. ${ }^{24}$ At $t=0$, we change the carrier frequency to $f_{\text {off-res }}$ far from $f_{\text {res}}$, as shown in Fig. 1(b). During this off-resonant time $t_{\text {pol }}$, the spin ensemble polarizes along the static magnetic field with a characteristic time $T_{1}$. By allowing the off-resonant condition to persist for a variety of different $t_{\text {pol }}$ before tuning the ARP pulses back onresonance and measuring the resulting $F$, we can measure the build-up of the ensemble's thermal polarization. In Fig. 2 , we show measurements of nanometer-scale ensembles of
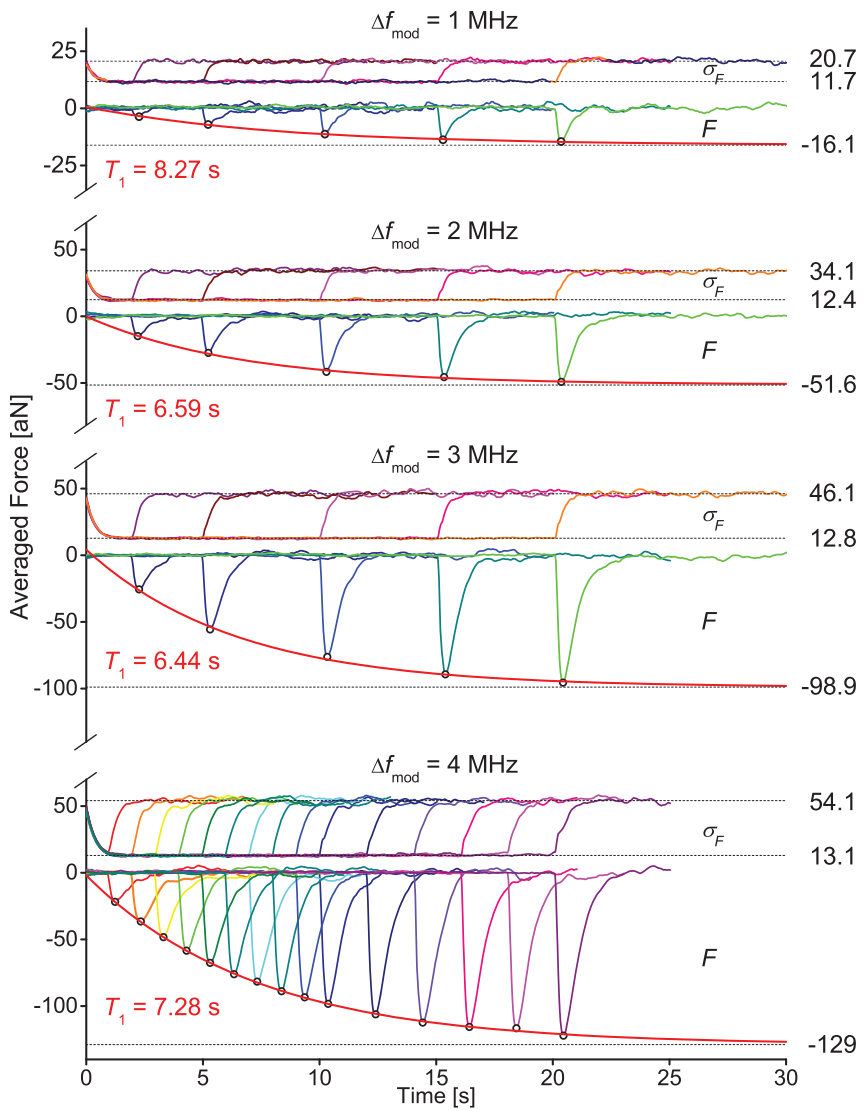

FIG. 2. $F$ and $\sigma_{F}$ averaged over 500 measurements at $B=4.37 \mathrm{~T}$ and $T=4.4 \mathrm{~K}$ for different modulation widths $\Delta f_{\bmod }=\{1,2,3,4\} \mathrm{MHz}$ of the ARP pulses. Each graph shows $F$ in the lower part, reflecting the thermal polarization, and $\sigma_{F}$ in the upper part, reflecting the statistical polarization, for a series of different polarization times $t_{\mathrm{pol}}$. Only at $t=t_{\mathrm{pol}}$ (black circles), when the ARP pulses are turned back on resonance, are $F$ and $\sigma_{F}$ related to the thermal and statistical spin polarization, respectively. During the polarization time $\left(t<t_{\mathrm{pol}}\right) \sigma_{F}=\sigma_{\text {cant }}$, while on resonance $\left(t>t_{\mathrm{pol}}\right)$ spin noise also contributes, i.e., $\sigma_{F}=\sqrt{\sigma_{\text {spin }}^{2}+\sigma_{\text {cant }}^{2}}$.

${ }^{19} \mathrm{~F}$ nuclear spins at $B=4.37 \mathrm{~T}$ and $T=4.4 \mathrm{~K}$. The on- and off-resonant carrier frequencies are $f_{\text {res }}=175 \mathrm{MHz}$ and $f_{\text {off- }}$ res $=168 \mathrm{MHz}$, respectively. Different ensemble sizes are addressed by changing the frequency modulation amplitude $\Delta f_{\text {mod }}$ of the ARP pulse sequences. The thickness of the resonant slice and therefore its volume of intersection with the sample is roughly proportional to $\Delta f_{\text {mod. }}$. From fits to these signals, as shown in Fig. 2, we can extract both the force $F$ due to the equilibrium thermal polarization and the spinlattice relaxation time $T_{1}$. By plotting the standard deviation of the resonant force $\sigma_{F}$ during the same experiments, we can also measure the effect of the ensemble's statistical fluctuations $\sigma_{M_{z}}$. As expected from Eqs. (1) and (2), for detection volumes with nearly constant $\frac{\partial B}{\partial x}, F$ increases linearly with increasing detection volume or roughly linearly with $\Delta f_{\text {mod }}$, while $\sigma_{\text {spin }}$ increases roughly as $\sqrt{\Delta f_{\text {mod }}}$.

From the ratio of the measured thermal and statistical polarizations and using Eq. (3) with $\frac{M_{z}}{\sigma_{M_{z}}}=\frac{F}{\sigma_{\text {spin }}}$, we determine the number of spins in the detected ensembles. $N$ ranges from $0.98 \times 10^{6}$ to $6.61 \times 10^{6}$ corresponding to detection volumes $V$ from $(26.3 \mathrm{~nm})^{3}$ to $(49.7 \mathrm{~nm})^{3}$. As shown in Fig. 3, pulses with the smallest $\Delta f_{\bmod }=1 \mathrm{MHz}$ address a spin ensemble slightly smaller than $N_{c}=1.10 \times 10^{6}$ spins, i.e. just small 


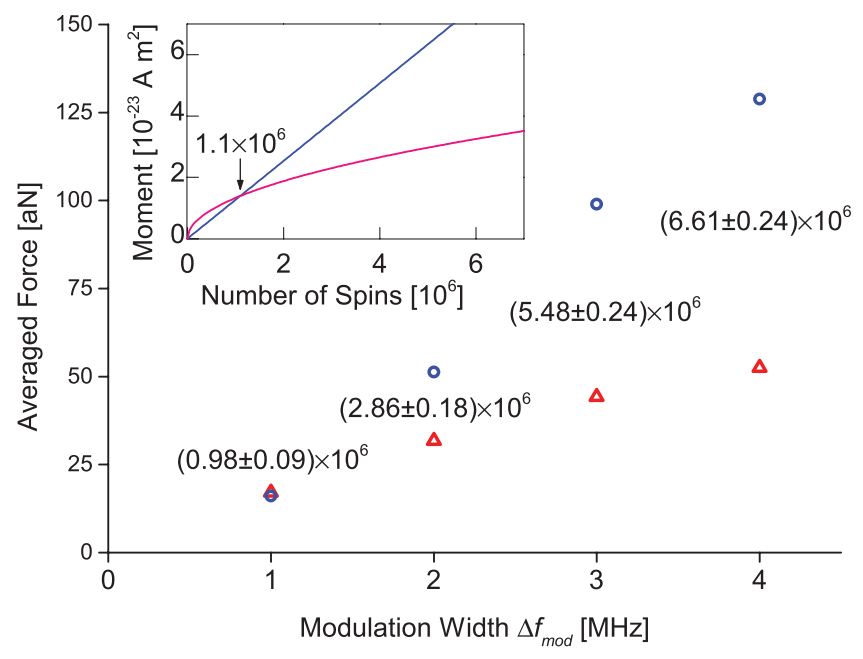

FIG. 3. $F$ (blue circles), originating from the thermal polarization, and $\sigma_{\text {spin }}$ (red triangles), originating from the statistical polarization, as a function of the ARP modulation width $\Delta f_{\bmod }$ at $B=4.37 \mathrm{~T}$ and $T=4.4 \mathrm{~K}$. The values between the symbols show the corresponding number of spins $N$ given by Eq. (3). Inset: A theoretical plot of $M_{z}$ and $\sigma_{M_{z}}$ for ${ }^{19} \mathrm{~F}$ as a function of $N$ showing the crossover at $N_{c}=1.10 \times 10^{6}$ spins. The similarity between the inset and the figure indicate that the number of detected spins (or the detection volume $V$ ) is roughly proportional to $\Delta f_{\text {mod. }}$.

enough to be dominated by statistical nuclear spin polarization. The calculated number of spins compare favorably to the lower limit of spins determined through estimates of the magnetic field gradient based on a magnetostatic model in the manner of the supplementary section of Peddibhotla et al. ${ }^{17}$

Experiments also show that $T_{1}=7.2 \pm 1.0 \mathrm{~s}$ and is independent of the ensemble size within the error of the measurement. This value is similar to previous measurements of larger detection volumes, yielding $T_{1}=6.2 \mathrm{~s}^{25}$ Precise comparisons are difficult given that $T_{1}$ depends strongly on the density of paramagnetic impurities in the sample as well as oxygen at the surface. Recent measurements of small ensembles of electron spins also show that a small detection volume can alter the measured $T_{1}$ relative to conventional measurements. For tiny detection volumes within a larger sample, the measured $T_{1}$ can be reduced by spin diffusion effects relative to measurements of macroscopic detection volumes. ${ }^{26}$

For detection volumes, in which $\frac{\partial B}{\partial x}$ is nearly constant, the error in the determination of the size of the detected ensemble depends only on the error of the measurements of $F$ and $\sigma_{F}$ and on the error in determining $B$ and $T$. This method therefore provides a complementary and, in some cases, more precise alternative to other techniques. In particular, in MRFM the size of the detected ensemble is usually determined by measuring $F$ or $\sigma_{F}$ (depending on whether the volume is in the thermal or statistical regime), estimating the magnetic field gradient, and calculating the number of moments responsible for the measured force. The precision of this scheme depends on knowledge of the magnetic field gradient at the sample and the spring constant of the cantilever. Often, such quantities are measured with a high degree of error. An estimate of the size of the detection volume can also be made through knowledge of the magnetic field profile of the tip, calculation of the resonant slice geometry, and knowledge of the shape and position of the sample. Again, such calculations are typically imprecise. In fact, our method can be applied to any NMR technique capable of detecting both the thermal and statistical polarizations. These include conventional RF probes at room temperature 27,28 and optical Faraday rotation methods in alkali metal vapors. ${ }^{29}$ In all cases, the comparison of statistical and thermal magnetization may provide additional information, especially when either the precise shape or density distribution of the sample is not known.

In conclusion, we perform NMR measurements of small ensembles of ${ }^{19} \mathrm{~F}$ nuclei showing the transition from a thermally dominated to a statistically dominated ensemble magnetization. In addition, we demonstrate a method for determining the number of spins in nanometer-scale ensembles by measuring and comparing both the thermal and statistical polarizations. These results are relevant to a number of recent experiments, which can now address nanometerscale ensembles of nuclear spins. Until today, statistical polarization in conventional NMR and MRI of macroscopic samples has played a limited role. ${ }^{30}$ The fact that even for a fairly large ensemble of $10^{6}{ }^{19} \mathrm{~F}$ nuclear spins at low temperature and high field natural polarization fluctuations overtake the thermal polarization underscores just how weak conventional NMR signals are. As methods for nanoMRI continue to develop, the role of statistical polarization, as highlighted here, will become increasingly important.

We acknowledge support from the Sino Swiss Science and Technology Cooperation (Project IZLCZ2 138894), the Swiss Nanoscience Institute (Project No. P1207), and the Swiss National Science Foundation (Grant No. 200020-140478).

${ }^{1}$ E. Frey and K. Kroy, Ann. Phys. 14, 20 (2005).

${ }^{2}$ B. J. van Wees, H. van Houten, C. W. J. Beenakker, J. G. Williamson, L. P. Kouwenhoven, D. van der Marel, and C. T. Foxon, Phys. Rev. Lett. 60, 848 (1988).

${ }^{3}$ L. Ciobanu, D. A. Seeber, and C. H. Pennington, J. Magn. Reson. 158, 178 (2002).

${ }^{4}$ J. A. Sidles, J. L. Garbini, K. J. Bruland, D. Rugar, O. Züger, S. Hoen, and C. S. Yannoni, Rev. Mod. Phys. 67, 249 (1995).

${ }^{5}$ C. L. Degen, M. Poggio, H. J. Mamin, C. T. Rettner, and D. Rugar, Proc. Natl. Acad. Sci. U.S.A. 106, 1313 (2009).

${ }^{6}$ H. J. Mamin, T. H. Oosterkamp, M. Poggio, C. L. Degen, C. T. Rettner, and D. Rugar, Nano Lett. 9, 3020 (2009).

${ }^{7}$ J. M. Nichol, T. R. Naibert, E. R. Hemesath, L. J. Lauhon, and R. Budakian, Phys. Rev. X 3, 031016 (2013).

${ }^{8}$ H. J. Mamin, M. Poggio, C. L. Degen, and D. Rugar, Nat. Nanotechnol. 2, 301 (2007).

${ }^{9}$ H. J. Mamin, M. Kim, M. H. Sherwood, C. T. Rettner, K. Ohno, D. D. Awschalom, and D. Rugar, Science 339, 557 (2013).

${ }^{10}$ T. Staudacher, F. Shi, S. Pezzagna, J. Meijer, J. Du, C. A. Meriles, F. Reinhard, and J. Wrachtrup, Science 339, 561 (2013).

${ }^{11}$ M. Loretz, S. Pezzagna, J. Meijer, and C. L. Degen, Appl. Phys. Lett. 104, 033102 (2014).

${ }^{12}$ D. Rugar, H. J. Mamin, M. H. Sherwood, M. Kim, C. T. Rettner, K. Ohno, and D. D. Awschalom, preprint arXiv:1406.2983.

${ }^{13}$ T. Häberle, D. Schmid-Lorch, F. Reinhard, and J. Wrachtrup, preprint arXiv:1406.3324.

${ }^{14}$ S. J. DeVience, L. M. Pham, I. Lovchinsky, A. O. Sushkov, N. Bar-Gill, C. Belthangady, F. Casola, M. Corbett, H. Zhang, M. Lukin, H. Park, A. Yacoby, and R. L. Walsworth, preprint arXiv:1406.3365.

${ }^{15}$ F. Bloch, Phys. Rev. 70, 460 (1946).

${ }^{16}$ C. Degen, M. Poggio, H. Mamin, and D. Rugar, Phys. Rev. Lett. 99, 250601 (2007).

${ }^{17}$ P. Peddibhotla, F. Xue, H. I. T. Hauge, S. Assali, E. P. A. M. Bakkers, and M. Poggio, Nat. Phys. 9, 631 (2013).

${ }^{18}$ F. Xue, D. P. Weber, P. Peddibhotla, and M. Poggio, Phys. Rev. B 84, 205328 (2011). 
${ }^{19}$ C. P. Schlichter, Principles of Magnetic Resonance, 3rd ed. (Springer, Berlin, 1996).

${ }^{20}$ M. Poggio, C. L. Degen, C. T. Rettner, H. J. Mamin, and D. Rugar, Appl. Phys. Lett. 90, 263111 (2007).

${ }^{21} \bar{E}$. Kupce and R. Freeman, J. Magn. Reson., Ser. A 118, 299 (1996).

${ }^{22}$ A. Tannús and M. Garwood, J. Magn. Reson., Ser. A 120, 133 (1996).

${ }^{23}$ In general, $F=\left\langle\frac{\partial B}{\partial x}\right\rangle_{V} M_{z}$ and $\sigma_{F}=\sqrt{\left\langle\left(\frac{\partial B}{\partial x}\right)^{2}\right\rangle_{V} \sigma_{M_{z}}^{2}+\sigma_{\text {cant }}^{2}}$, where $\langle\cdots\rangle_{V}$ denotes an average taken over the detection volume $V$. For detection volumes small enough that $\frac{\partial B}{\partial x}$ is slowly varying throughout, as in all experiments discussed here, $\left(\left\langle\frac{\partial B}{\partial x}\right\rangle_{V}\right)^{2}=\left\langle\left(\frac{\partial B}{\partial x}\right)^{2}\right\rangle_{V}$.
${ }^{24}$ C. L. Degen, M. Poggio, H. J. Mamin, and D. Rugar, Phys. Rev. Lett. 100, 137601 (2008).

${ }^{25}$ K. Eberhardt, C. L. Degen, and B. Meier, Phys. Rev. B 76, 180405 (2007).

${ }^{26}$ J. Cardellino, N. Scozzaro, M. Herman, A. J. Berger, C. Zhang, K. C. Fong, C. Jayaprakash, D. V. Pelekhov, and P. C. Hammel, Nat. Nanotechnol. 9, 343 (2014).

${ }^{27}$ M. McCoy and R. Ernst, Chem. Phys. Lett. 159, 587 (1989).

${ }^{28}$ M. Guéron and J. L. Leroy, J. Magn. Reson. 85, 209 (1989).

${ }^{29}$ S. A. Crooker, D. G. Rickel, A. V. Balatsky, and D. L. Smith, Nature 431, 49 (2004).

${ }^{30}$ N. Müller and A. Jerschow, Proc. Natl. Acad. Sci. U.S.A. 103, 6790 (2006). 